\title{
Path Selection and Rate Allocation in Self-Backhauled mmWave Networks
}

\author{
Trung Kien $\mathrm{Vu}^{*}$, Chen-Feng Liu*, Mehdi Bennis*, Mérouane Debbah ${ }^{\dagger \ddagger}$, and Matti Latva-aho* \\ ${ }^{*}$ Centre for Wireless Communications, University of Oulu, Finland \\ ${ }^{\dagger}$ Mathematical and Algorithmic Sciences Lab, Huawei France R\&D, Paris, France \\ ${ }_{\ddagger}$ CentraleSupelec, Universite Paris-Saclay, Gif-sur-Yvette, France \\ E-mail: \{trungkien.vu, chen-feng.liu, mehdi.bennis, matti.latva-aho\}@oulu.fi, merouane.debbah@ huawei.com
}

\begin{abstract}
We investigate the problem of multi-hop scheduling in self-backhauled millimeter wave (mmWave) networks ${ }^{1}$. Owing to the high path loss and blockage of mmWave links, multi-hop paths/routes between the macro base station and the intended users via full-duplex small cells need to be carefully selected. This paper addresses the fundamental question: "how to select the best paths and how to allocate rates over these paths subject to latency constraints?" To answer these questions, we propose a new system design, which factors in mmWave-specific channel variations and network dynamics. The problem is cast as a network utility maximization subject to a bounded delay constraint and network stability. The studied problem is decoupled into: $(i)$ a path/route selection and $(i i)$ rate allocation, whereby learning the best paths is done by means of a reinforcement learning algorithm, and the rate allocation is solved by applying the successive convex approximation method. Via numerical results, our approach ensures reliable communication with a guaranteed probability of $99.9999 \%$, and reduces latency by $50.64 \%$ and $92.9 \%$ as compared to baselines.
\end{abstract}

Index Terms-URLLC, low latency, reliable communication, mmWave communications, multi-hop scheduling, ultra dense small cells, stochastic optimization, reinforcement learning, nonconvex optimization.

\section{INTRODUCTION}

The fifth generation $(5 \mathrm{G})$ networks are required to support high data rates of multiple gigabits per second (Gbps) and to have 50 billion connected devices by 2020 [1]. In parallel to that, due to the current scarcity of wireless spectrum, both academia and industry have paid attention to the underutilized frequency bands (30-300 GHz) [1], [2]. The required capacity increase can be achieved by $(i)$ advanced spectral-efficient transmission techniques, e.g., massive multiple-input multipleoutput (MIMO); and (ii) ultra-dense self-backhauled small cell deployments [3], [4]. Although mmWave frequency bands offer huge bandwidth, operating at higher frequency bands experiences high propagation attenuation [2], which requires smart beamforming to achieve highly directional gains. Owing to the short wavelength, mmWave frequency bands enable packing a massive number of antennas into highly directional beamforming over a short distance as compared to the conventional frequency bands [2]. Besides that, mmWave communication requires higher transmit power and is very sensitive to blockage, when transmitting over a long distance

${ }^{1}$ This paper was presented at the IEEE WCNC 2018 Conference, MAC9 mmWave MAC Design, in Barcelona, Catalonia, Spain, April 18, 2018.
[2], [4]. Hence, instead of using a single hop [4], [5], a multihop self-backhauling architecture is a promising solution [6], [7].

Focusing on maximizing the quality of multimedia applications, the authors in [8] studied multi-hop routing for deviceto-device communication. The work [9] studied the multihop relaying transmission challenges for mmWave systems. Therein, taking traffic dynamics and link qualities into account, [8], [9] aimed at maximizing the network throughput. In addition, path selection and multi-path congestion control was studied in [10] in which the aggregate utility is increased as more paths are provided.

Despite the interesting results of the aforementioned works, using multi-hop transmissions raises the issue of increased delay which has been generally ignored. Note that the issues of latency and reliability are two key components in 5G networks and beyond [11]. Moreover, splitting data into too many paths leads to increased signaling overhead and causes network congestion. Hence, there is a need for fast and efficient multihop multi-path scheduling with respect to traffic dynamics and channel fluctuations in self-backhauled mmWave networks. Our previous studies focused on single-hop ultra-reliable low latency communication (URLLC)-centric transmission in mmWave networks [5]. In this work, we further extend the previous work to the multi-hop multi-path wireless backhaul scenario and study a joint path selection and rate allocation problem. In summary, we address two fundamental aspects enabling multi-hop multi-path self-backhauled mmWave networks: $(i)$ how to select the best paths while taking traffic dynamics and link qualities into account; (ii) how to capture elements of URLLC while maximizing the network utility.

\section{A. Main contribution}

Considering a multi-hop multi-path self-backhauled mmWave network, we propose an efficient system design to support URLLC. In particular, our goal is to maximize a general network utility subject to network stability and the delay bound violation constraint with a tolerable probability (reliability). Leveraging Lyapunov stochastic optimization [12], the studied problem is decoupled into multi-hop path/route selection and rate allocation sub-problems. The challenging questions we seek to address are: $(i)$ over which paths should the traffic flow be forwarded? and (ii) what is 
TABLE I: Notations for system model.

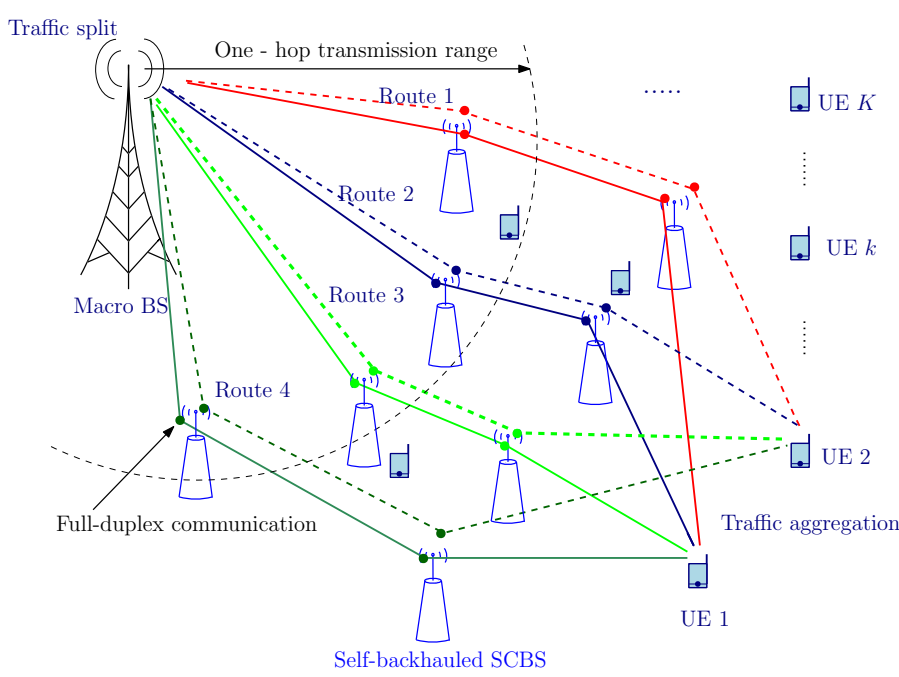

Fig. 1: Illustration of $5 \mathrm{G}$ multi-hop self-backhauled mmWave networks.

the data rate per flow/sub-flow while ensuring low-latency and ultra-reliability constraints? To answer these questions, we utilize regret learning techniques to exploit the benefits of the historical information which aids in selecting the best paths. For rate allocation, the corresponding mathematical problem belongs to a non-convex combinatorial program [13]. By exploiting the hidden convexity of the problem, we propose an iterative rate allocation algorithm based on the second-order cone program (SOCP) to obtain a local optimal of the approximated convex problem. Numerical results verify the effectiveness of the proposed path selection and rate allocation solution.

\section{System ModeL}

Let us consider a downlink (DL) transmission of a multihop heterogeneous cellular network $(\mathrm{HCN})$ which consists of a macro base station (MBS), a set of $B$ self-backhauled small cell base stations (SCBSs), and a set $\mathcal{K}$ of $K$ singleantenna user equipments (UEs) as shown in Fig 1. Let $\mathcal{B}=\{0,1, \cdots, B\}$ denote the set of all base stations (BSs) in which index 0 refers to the MBS. The in-band wireless backhaul is used to provide backhaul among BSs [14]. A full-duplex (FD) transmission protocol is assumed at SCBS capable with perfect self-interference cancellation (SIC) capabilities. Each BS is equipped with $N_{b}$ transmitting antennas and we denote the propagation channel between BS $b$ and UE $k$ as $\mathbf{h}_{(b, k)}=\sqrt{N_{b}} \Theta_{(b, k)}^{1 / 2} \mathbf{w}_{(b, k)}$ [4], where $\boldsymbol{\Theta}_{(b, k)} \in \mathbb{C}^{N_{b} \times N_{b}}$ depicts the antenna spatial correlation, and the elements of $\mathbf{w}_{(b, k)} \in \mathbb{C}^{N_{b} \times 1}$ are independent and identically distributed (i.i.d.) with zero mean and variance $1 / N_{b}$.

The network topology is modeled as a directed graph $\mathcal{G}=(\mathcal{N}, \mathcal{L})$, where $\mathcal{N}=\mathcal{B} \cup \mathcal{K}$ represents the set of nodes including BSs and UEs. $\mathcal{L}=\{(i, j) \mid i \in \mathcal{B}, j \in \mathcal{N}\}$ denotes the set of all directional edges $(i, j)$ in which nodes $i$ and $j$ are the transmitter and the receiver, respectively.

We consider a queuing network operating in discrete time $t \in \mathbb{Z}^{+}$. There are $F$ independent data at the MBS. Each

\begin{tabular}{|c|l|}
\hline Notations & Descriptions \\
\hline \hline $\mathcal{B}, \mathcal{K}$ & Sets of $(B+1)$ base stations, $K$ user equipments \\
\hline $\mathcal{N}=\mathcal{B} \cup \mathcal{K}$ & Set of nodes including BSs and UEs \\
\hline $\mathcal{L}$ & Set of all directional edges $(i, j) \mid i \in \mathcal{B}, j \in \mathcal{N}$ \\
\hline $\mathcal{F}$ & Set of $F$ flows \\
\hline $\mathcal{Z}_{f}$ & Set of $Z_{f}$ disjoint paths observed by flow $f$ \\
\hline $\mathcal{N}_{i}^{(\mathrm{o})}$ & Set of the next hops from node $i$ \\
\hline$i_{f}^{(\mathrm{I})}$ & Previous hop of flow $f$ to BS $i$ \\
\hline$i_{f}^{(\mathrm{o})}$ & Next hop of flow $f$ from BS $i$ \\
\hline$p_{(i, j)}^{f}$ & Transmit power of node $i$ to node $j$ for flow $f$ \\
\hline$z_{f}^{m}=1$ & Path $m$ is used to send data for flow $f$ \\
\hline$\pi_{f}^{m}$ & Probability of choosing path $m$ for flow $f$ \\
\hline
\end{tabular}

data traffic is destined for only one UE, whereas one UE can receive multiple data streams, i.e., $F \geq K$. Hereafter, we refer to data traffic as data flow. We use $\mathcal{F}$ to represent the set of $F$ data flows/sub-flows. The MBS can split each flow $f \in$ $\mathcal{F}$ into multiple sub-flows which are sent through a set of disjoint paths. The traffic aggregation capability is assumed at the UEs [15].

We assume that there exits $Z_{f}$ number of disjoint paths from the MBS to the UE for flow $f$. For any disjoint path $m \in\left\{1, \cdots, Z_{f}\right\}$, we denote $\mathcal{Z}_{f}^{m}$ as the path state, which contains all path information such as topology and queue states for every hop. Let $\mathcal{Z}_{f}=\left\{\mathcal{Z}_{f}^{1}, \cdots, \mathcal{Z}_{f}^{m}, \cdots, \mathcal{Z}_{f}^{Z_{f}}\right\}$ denote path states observed by flow $f$. We use the flow-split indicator vector $\mathbf{z}_{f}=\left(z_{f}^{1}, \cdots, z_{f}^{Z_{f}}\right)$ to denote how the MBS splits flow $f$, where $z_{f}^{m}=1$ means path $m$ is used to send data for flow $f$; otherwise, $z_{f}^{m}=0$. Let $\mathcal{N}_{i}^{(\mathrm{o})}$ denote the set of the next hops from node $i$ via a directional edge. We denote the next hop and the previous hop of flow $f$ from and to BS $i$ as $i_{f}^{(\mathrm{o})}$ and $i_{f}^{(\mathrm{I})}$, respectively. Table I shows the notations, which used through this paper.

In addition, $\mathbf{h}=\left(\mathbf{h}_{(i, j)} \mid(i, j) \in \mathcal{L}\right)$ is the channel propagation vector, and we denote $p_{(i, j)}^{f}$ as the transmit power of node $i$ assigned to node $j$ for flow $f$, such that $\sum_{f \in F} \sum_{j \in \mathcal{N}_{i}^{(\circ)}} p_{(i, j)}^{f} \leq P_{i}^{\max }$, where $P_{i}^{\max }$ is the maximum transmit power of node $i$. We have the power constraint as

$$
\mathcal{P}=\left\{p_{(i, j)}^{f} \geq 0, i, j \in \mathcal{N}, \mid \sum_{f \in \mathcal{F}} \sum_{j \in \mathcal{N}_{i}^{(o)}} p_{(i, j)}^{f} \leq P_{i}^{\max }\right\}
$$

Vector $\mathbf{p}=\left(p_{(i, j)}^{f} \mid \forall i, j \in \mathcal{N}, \forall f \in \mathcal{F}\right)$ denotes the transmit power over all flows.

Here, we assume that each BS adopts the hybrid beamforming architecture, which enjoys both analog and digital beamforming techniques [16]. For the analog beamforming, let $g_{(i, j)}^{(t)}$ and $g_{(i, j)}^{(r)}$ denote the transmitter and receiver beamforming gain at the transmitter $i$ and the receiver $j$, respectively. In addition, we use $\omega_{(i, j)}^{(t)}$ and $\omega_{(i, j)}^{(r)}$ to represent the angles deviating from the strongest path between the transmitter $i$ and the receiver $j$. Also, let $\theta_{(i, j)}^{(t)}$ and $\theta_{(i, j)}^{(r)}$ denote the beamwidth at the transmitter $i$ and the receiver $j$, respectively. We denote $\boldsymbol{\theta}$ as a vector of the transmitter beamwidth of all BSs. We adapt the widely used antenna radiation pattern model [16], [17] to determine the beamforming gain as 


\section{Problem Formulation}

$g_{(i, j)}\left(\omega_{(i, j)}, \theta_{(i, j)}\right)= \begin{cases}\frac{2 \pi-\left(2 \pi-\theta_{(i, j)}\right) \eta}{\theta_{(i, j)}}, & \text { if }\left|\omega_{(i, j)}\right| \leq \frac{\theta_{(i, j)}}{2}, \\ \eta, & \text { otherwise, }\end{cases}$ where $0<\eta \ll 1$ is the side lobe gain. For the digital beamforming phase, we apply the linear precoding scheme $\mathbf{v}_{(i, j)}$, i.e., for the conjugate precoding, $\mathbf{v}\left(\mathbf{h}_{(i, j)}\right)=\hat{\mathbf{h}}_{(i, j)}$. Here, $\hat{\mathbf{h}}_{(i, j)}$ is the estimated channel of $\mathbf{h}_{(i, j)}$, such that

$$
\hat{\mathbf{h}}_{(i, j)}=\sqrt{N_{i}} \Theta_{(i, j)}^{1 / 2}\left(\sqrt{1-\tau_{j}^{2}} \mathbf{w}_{(i, j)}+\tau_{j} \hat{\mathbf{w}}_{(i, j)}\right),
$$

where $\tau_{j} \in[0,1]$ reflects the estimation accuracy for receiver $j$, if $\tau_{j}=0$, the perfect channel state information is assumed at the transmitters [18]. $\hat{\mathbf{w}}_{(i, j)} \in \mathbb{C}^{N_{i}}$ is the estimated noise vector, also modeled as a random matrix with zero mean and variance of $\frac{1}{N_{i}}$ [4]. Based on the hybrid model [16], the Ergodic achievable rate $^{2}$ of and the receiver $j$ from the transmitter $i$ can be calculated as per (2), where $p_{(i, j)}$ and $p_{\left(i^{\prime}, j\right)}$ are the transmit power from the transmitter $i$ and $i^{\prime}$ to the receiver $j$, respectively, and the thermal noise of receiver $j$ is $\eta_{(i, j)}$. In addition, $\mathrm{W}$ denotes the system bandwidth of the mmWave frequency band.

Therefore, for a given channel state and transmit power, the data rate in edge $(i, j)$ over flow $f$ can be posted as a function of channel state and transmit power, i.e., $R_{f}^{(i, j)}(\mathbf{h}, \mathbf{p})$, such that $\sum_{f \in \mathcal{F}} R_{f}^{(i, j)}=R^{(i, j)}$. We denote $\mathbf{R}=\left(R_{f}^{(i, j)} \mid \forall i, j \in\right.$ $\mathcal{N}, \forall f \in \mathcal{F})$ as a vector of data rates over all flows.

Let $Q_{f}^{i}(t)$ denote the queue length at BS $i$ at time slot $t$ for flow $f$. The queue length evolution at the MBS $i=0$ is

$Q_{f}^{i}(t+1)=\left[Q_{f}^{i}(t)-\sum_{m=1, i_{f}^{(\mathrm{o})} \in \mathcal{N}_{i}^{(\mathrm{o})}}^{Z_{f}} R_{f}^{\left(i, i_{f}^{(\mathrm{o})}\right)}(t), 0\right]^{+}+\mu^{f}(t)$,

where $\mu^{f}(t)$ is the data arrival at the MBS during slot $t$, which is independent and identical distributed (i.i.d.) over time with a mean value $\bar{\mu}^{f}$. Due to the disjoint paths, for each flow $f$ the incoming rate from the previous hop $i_{f}^{(\mathrm{I})}$ at the SCBS $i$ is either from another SCBS or the MBS, and thus, the queue evolution at the SCBS $i=\{1, \cdots, B\}$ is given by

$$
Q_{f}^{i}(t+1) \leq\left[Q_{f}^{i}(t)-R_{f}^{\left(i, i_{f}^{(\mathrm{o})}\right)}(t), 0\right]^{+}+R_{f}^{\left(i_{f}^{(\mathrm{I})}, i\right)}(t) .
$$

Definition 1. For any vector $\mathbf{x}(t)=\left(x_{1}(t), \ldots, x_{K}(t)\right)$, let $\overline{\mathbf{x}}=\left(\bar{x}_{1}, \cdots, \bar{x}_{K}\right)$ denote the time average expectation of $\mathbf{x}(t)$, where $\overline{\mathbf{x}} \triangleq \lim _{t \rightarrow \infty} \frac{1}{t} \sum_{\tau=0}^{t-1} \mathbb{E}[\mathbf{x}(\tau)]$.

Definition 2. For any discrete queue $Q(t)$ over time slots $t \in\{0,1, \ldots\}$ and $Q(t) \in R_{+}$,

- $Q(t) \quad$ is $\quad$ strongly
$\lim _{t \rightarrow \infty} \sup \frac{1}{t} \sum_{\tau=0}^{t-1} \mathbb{E}[|Q(\tau)|]<\infty$. stable
- $Q(t)$ is mean rate stable if $\lim _{t \rightarrow \infty} \frac{\mathbb{E}[|Q(t)|]}{t}=0$.

A queue network is stable if each queue is stable.

${ }^{2}$ Note that we omit the beam search/track time, since it can be done fast and is very small as compared the transmission time [19].
Assume that the MBS determines paths to split data flow $f$ with a given probability distribution, i.e., $\pi_{f}=$ $\left(\pi_{f}^{1}, \cdots, \pi_{f}^{Z_{f}}\right)$, where for each $m \in \mathcal{Z}_{f}$ we have $\pi_{f}^{m}=$ $\operatorname{Pr}\left(z_{f}=z_{f}^{m}\right)$. Here, $\boldsymbol{\pi}_{f}$ is the probability mass function (PMF) of the flow-split vector, i.e., $\sum_{m=1}^{Z_{f}} \operatorname{Pr}\left(z_{f}^{m}\right)=1$. We denote $\boldsymbol{\pi}=\left\{\boldsymbol{\pi}_{1}, \cdots, \boldsymbol{\pi}_{f}, \cdots, \boldsymbol{\pi}_{F}\right\} \in \Pi$ as the global probability distribution of all flow-split vectors in which $\Pi$ is the set of all possible global PMFs. Let $\bar{x}_{f}$ denote the achievable average rate of flow $f$, where $\bar{x}_{f} \triangleq \lim _{t \rightarrow \infty} \frac{1}{t} \sum_{\tau=0}^{t-1} x_{f}(\tau)$ and $x_{f}(\tau)=\left.\sum_{m=1, i_{f}^{(\circ)} \in \mathcal{N}_{i}^{(o)}}^{Z_{f}} \mathbb{E}_{\mathbf{h}, \mathbf{p}}\left[\pi_{f}^{m} R_{f}^{\left(i, i_{f}^{(o)}\right)}(\tau)\right]\right|_{i=0}$. We assume that the achievable rate is bounded, i.e.,

$$
0 \leq x_{f}(t) \leq a_{f}^{\max }
$$

where $a_{f}^{\max }$ is the maximum achievable rate of flow $f$ at every time $t$. Vector $\overline{\mathrm{x}}=\left(\bar{x}_{1}, \cdots, \bar{x}_{F}\right)$ denotes the time average of rates over all flows. Let $\mathcal{R}$ denote the rate region, which is defined as the convex hull of the average rates, i.e., $\overline{\mathrm{x}} \in \mathcal{R}$.

We define $U_{0}$ as a network utility function, i.e., $U_{0}(\overline{\mathbf{x}})=$ $\sum_{f \in \mathcal{F}} U\left(\bar{x}_{f}\right)$. Here, $U(\cdot)$ is assumed to be a twice differentiable, concave, and increasing $L$-Lipschitz function for all $\overline{\mathbf{x}} \geq 0$. According to Little's law [20], the queuing delay is defined as the ratio of the queue length to the average arrival rate. By taking into account the probabilistic delay constraints for each flow/subflow, the following network utility maximization (NUM) is formulated as:

$$
\begin{array}{ll}
\text { OP: } \max _{\boldsymbol{\pi}, \mathbf{x}, \mathbf{p}} & U_{0}(\overline{\mathbf{x}}) \\
\text { subject to } & \operatorname{Pr}\left(\frac{Q_{f}^{i}(t)}{\bar{\mu}_{f}} \geq \beta\right) \leq \epsilon, \forall t, f \in \mathcal{F}, i \in \mathcal{B}, \\
& \lim _{t \rightarrow \infty} \frac{\mathbb{E}\left[\left|Q_{f}^{i}\right|\right]}{t}=0, \forall f \in \mathcal{F}, \forall i \in \mathcal{B}, \\
& \mathbf{x}(t) \in \mathcal{R}, \\
& \pi \in \Pi, \\
& \text { and (1), (5), }
\end{array}
$$

where $\operatorname{Pr}(\cdot)$ denotes the probability operator, $\beta$ reflects the maximum allowed delay requirement for UEs, and $\epsilon \ll 1$ is the target probability for reliable communication. The probabilistic delay constraint (6b) implies that the probability that the delay for each flow at node $b$ is greater than $\beta$ is very small, which captures the constraints of ultra-low latency and reliable communication. It is also used to avoid congestion for each flow $f$ at any point (BS) in the network, if the queue length is greater than $\beta \bar{\mu}^{f}$. More importantly, (6b) forces the transmission of all BSs, and (6c) maintains network stability.

The above problem has a non-linear probabilistic constraint (6b), which cannot be solved directly. Hence, we replace the non-linear constraint (6b) with a linear deterministic equivalent by applying Markov's inequality [21], [5] such that 


$$
R^{(i, j)}=\mathbb{E}_{\mathbf{h}, \mathbf{p}}\left[\mathrm{W} \log _{2}\left(1+\frac{p_{(i, j)} g_{(i, j)}^{(t)} g_{(i, j)}^{(r)}\left|\mathbf{h}_{(i, j)}^{\dagger} \mathbf{v}_{(i, j)}\right|^{2}}{\sum_{i^{\prime} \neq i} p_{\left(i^{\prime}, j\right)} g_{\left(i^{\prime}, j\right)}^{(t)} g_{\left(i^{\prime}, j\right)}^{(r)}\left|\mathbf{h}_{\left(i^{\prime}, j\right)}^{\dagger} \mathbf{v}_{\left(i^{\prime}, j\right)}\right|^{2}+\eta_{(i, j)}}\right)\right]
$$

$\operatorname{Pr}(X \geq a) \leq \mathbb{E}[X] / a$ for a non-negative random variable $X$ and $a>0$. Thus, we relax (6b) as

$$
\mathbb{E}\left[Q_{f}^{i}(t)\right] \leq \bar{\mu}^{f} \epsilon \beta .
$$

Assuming that $\mu^{f}(t)$ follows a Poisson arrival process [21], we derive the expected queue length in (3) for $i=0$ as

$$
\mathbb{E}\left[Q_{f}^{i}(t)\right]=t \bar{\mu}^{f}-\sum_{\tau=1}^{t} \sum_{m=1, i_{f}^{(\circ)} \in \mathcal{N}_{i}^{(\circ)}} \pi_{f}^{m} R_{f}^{\left(i, i_{f}^{(\circ)}\right)}(\tau),
$$

and the expected queue length in (4), for each SCBS, i.e.,

$$
\mathbb{E}\left[Q_{f}^{i}(t)\right]=\sum_{\tau=1}^{t} \sum_{m} \pi_{f}^{m}\left(R_{f}^{\left(i_{f}^{(\mathrm{I})}, i\right)}(\tau)-R_{f}^{\left(i, i_{f}^{(\circ)}\right)}(\tau)\right) .
$$

Subsequently, combining the constraints (7) and (8), we obtain, for MBS $i=0$,

$$
\begin{aligned}
& \bar{\mu}^{f}(t-\epsilon \beta)-\sum_{\tau=1}^{t-1} \sum_{m=1, i_{f}^{(\circ)} \in \mathcal{N}_{i}^{(\circ)}} \pi_{f}^{m} R_{f}^{\left(i, i_{f}^{(\circ)}\right)}(\tau) \\
& \leq \sum_{m=1, i_{f}^{(\circ)} \in \mathcal{N}_{i}^{(\circ)}} \pi_{f}^{m} R_{f}^{\left(i, i_{f}^{(\circ)}\right)}(t) .
\end{aligned}
$$

Similarly, for each SCBS $i=\{1, \cdots, B\}$, we have

$$
\begin{aligned}
& -\bar{\mu}^{f} \epsilon \beta+\sum_{\tau=1}^{t-1} \sum_{m} \pi_{f}^{m}\left(R_{f}^{\left(i_{f}^{(\mathrm{I})}, i\right)}(\tau)-R_{f}^{\left(i, i_{f}^{(\circ)}\right)}(\tau)\right) \\
& \leq \sum_{m} \pi_{f}^{m}\left(R_{f}^{\left(i, i_{f}^{(\circ)}\right)}(t)-R_{f}^{\left(i_{f}^{(\mathrm{I})}, i\right)}(t)\right),
\end{aligned}
$$

by combining (7) and (9). With the aid of the above derivations, we consider (10) and (11) instead of (6b) in the original problem (6). In practice, the statistical information of all candidate paths to decide $\boldsymbol{\pi}_{f}, \forall f \in \mathcal{F}$, is not available beforehand, and thus solving (6) is very difficult. One solution is that paths are randomly assigned to each flow which does not guarantee optimality, whereas applying an exhaustive search is not practical. Therefore, in this work, we propose a low-complexity approach by invoking tools from Lyapunov stochastic optimization which achieves the optimal performance without requiring the statistical information beforehand.

\section{Proposed Algorithm}

In this section, we propose a Lyapunov optimization based framework in order to solve (6). To do that, we first introduction the auxiliary variables to refine the original problem (6). Next, we convert the constraints into virtual queues and write the conditional Lyapunov drift function. Finally, the solution of equivalent problem is obtained by minimizing the Lyapunov drift and the penalty from the objective function.

Let us start by rewriting (6) equivalently as [22]

$$
\begin{array}{ll}
\text { RP: } \max _{\bar{\varphi}, \boldsymbol{\pi}, \mathbf{p}} & U_{0}(\overline{\boldsymbol{\varphi}}) \\
\text { subject to } & \overline{\varphi_{f}}-\overline{x_{f}} \leq 0, \forall f \in \mathcal{F},
\end{array}
$$

$$
\text { (1), (5), (6c), (6e), (10), (11), }
$$

where the new constraint (12b) is introduced to replace the rate constraint (6d) with new auxiliary variables $\varphi=$ $\left(\varphi_{1}, \cdots, \varphi_{F}\right)$. In (12b), $\bar{\varphi} \triangleq \lim _{t \rightarrow \infty} \frac{1}{t} \sum_{\tau=0}^{t-1} \mathbb{E}[|\varphi(\tau)|]$. In order to ensure the inequality constraint (12b), we introduce a virtual queue vector $Y_{f}(t)$, which is given by

$$
Y_{f}(t+1)=\left[Y_{f}(t)+\varphi_{f}(t)-x_{f}(t)\right]^{+}, \forall f \in \mathcal{F} .
$$

Let $\boldsymbol{\Sigma}(t)=(\mathbf{Q}(t), \mathbf{Y}(t))$ denote the queue backlogs, we first write the conditional Lyapunov drift for slot $t$ as

$$
\boldsymbol{\Delta}(\boldsymbol{\Sigma}(t))=\mathbb{E}[L(\boldsymbol{\Sigma}(t+1))-L(\boldsymbol{\Sigma}(t)) \mid \boldsymbol{\Sigma}(t)],
$$

where $L(\boldsymbol{\Sigma}(t)) \triangleq \frac{1}{2}\left[\sum_{f=1}^{F} \sum_{i=0}^{B} Q_{f}^{i}(t)^{2}+\sum_{f=1}^{F} Y_{f}(t)^{2}\right]$ is the quadratic Lyapunov function of $\boldsymbol{\Sigma}(t)$ [12]. We then apply the Lyapunov drift-plus-penalty technique [12], [22], [4], where the solution of (12) is obtained by minimizing the Lyapunov drift and a penalty from the objective function, i.e.,

$$
\min \quad \boldsymbol{\Delta}(\boldsymbol{\Sigma}(t))-\nu \mathbb{E}\left[U_{0}(\bar{\varphi}) \mid \boldsymbol{\Sigma}(t)\right] .
$$

Here, $\nu$ is a control parameter to trade off utility optimality and queue length [4]. Note that the stability of $\boldsymbol{\Sigma}(t)$ assures that the constraints of problem (6c) and (12b) are held. Subsequently, following the straightforward calculations of the Lyapunov optimization which are omitted here for space, assuming that $\varphi \in \mathcal{R}$ and a feasible $\pi$ and all possible $\boldsymbol{\Sigma}(t)$ for all $t$, we obtain

$$
\begin{aligned}
\leq & \sum_{f=1}^{F} \sum_{i=1}^{B} Q_{f}^{i} \mathbb{E}\left[\sum_{m} \pi_{f}^{m}\left(R_{\left(i_{f}^{(\mathrm{I})}, i\right)}^{f}-R_{\left(i, i_{f}^{(\circ)}\right)}^{f}\right) \mid \boldsymbol{\Sigma}(t)\right] \\
& -\sum_{f=1}^{F} Q_{f}^{i \mid i=0} \mathbb{E}\left[\sum_{m=1, i_{f}^{(\circ)} \in \mathcal{N}_{i}^{(\circ)}} \pi_{f}^{m} R_{f}^{\left(i, i_{f}^{(\circ)}\right)} \mid \boldsymbol{\Sigma}(t)\right](16) \\
& +\sum_{f=1}^{F} \mathbb{E}\left[Y_{f} \varphi_{f}-\nu U\left(\varphi_{f}\right)-Y_{f} x_{f} \mid \boldsymbol{\Sigma}(t)\right]+\Psi .
\end{aligned}
$$

Here, the constant value $\Psi$ does not influence the system performance [12], [4]. The solution to (12) can be obtained by minimizing the upper bound in (16). For every slot $t$, we observe $\boldsymbol{\Sigma}(t)$ and have three decoupled subproblems as follows: The flow-split vector and the probability distribution are determined by 


$$
\text { SP1 : } \min _{\pi} \quad \sum_{f=1}^{F} \Xi_{f}
$$

subject to (6e),

where

$$
\begin{aligned}
\Xi_{f}= & \sum_{i=1}^{B} Q_{f}^{i} \sum_{m} \pi_{f}^{m}\left(R_{f}^{\left(i_{f}^{(\mathrm{I})}, i\right)}-R_{f}^{\left(i, i_{f}^{(\circ)}\right)}\right) \\
& -Q_{f}^{i \mid i=0} \sum_{m=1, i_{f}^{(\circ)} \in \mathcal{N}_{i}^{(\circ)}} \pi_{f}^{m} R_{\left(i, i_{f}^{(\circ)}\right)}^{f}
\end{aligned}
$$

Then, we select the optimal auxiliary variables by solving the following convex optimization problem

$$
\begin{array}{ll}
\text { SP2: } \min _{\varphi \mid \boldsymbol{\pi}} & \sum_{f=1}^{F}\left[Y_{f} \varphi_{f}-\nu U\left(\varphi_{f}\right)\right] \\
\text { subject to } & \varphi_{f}(t) \geq 0, \forall f \in \mathcal{F} .
\end{array}
$$

Let $\varphi_{f}^{*}$ be the optimal solution obtained by the first order derivative of the objective function of SP2. Assuming a logarithmic utility function, we have $\varphi_{f}^{*}(t)=\max \left\{\frac{\nu}{Y_{f}}, 0\right\}$. Finally, the rate allocation is done by assigning transmit power which is obtained by

$$
\begin{aligned}
\text { SP3: } \min _{\mathbf{x}, \mathbf{p} \mid \boldsymbol{\pi}} & \sum_{f=1}^{F}-Y_{f} x_{f} \\
\text { subject to } & (1),(5),(10),(11) .
\end{aligned}
$$

\section{A. Path Selection}

Now we leverage regret learning which exploits the historical system information such as queue state and channel state to select the optimal paths in SP1 [23]. The intuition behind this approach is that the regret learning method results in maximizing the long-term utility for each flow. Recall that $\mathbf{z}_{f}$ represents the flow-split vector given to flow $f$ and $z_{f}^{m}=1$ means path $m$ is used to send data for flow $f$. The MBS selects paths for each flow with a given probability (mixed strategy). The optimal strategies mean that the MBS does not wish to change its strategy for any flow where any deviation does not offer better utility gain for all flows. We denote $u_{f}^{m}=u_{f}\left(z_{f}^{m}, \mathbf{z}_{f}^{-m}\right)$ as a utility function of flow $f$ when using path $m$. The vector $\mathbf{z}_{f}^{-m}$ denotes the flow-split vector excluding path $m$. The MBS can choose more than one path to deliver data, from SP1, the utility gain of flow $f$ is

$$
u_{f}=\sum_{m} u_{f}^{m}=-\Xi_{f} .
$$

To exploit the historical information, the MBS determines a flow-split vector for each flow $f$ from $\mathcal{Z}_{f}$ based on the PMF from the previous stage $t-1$, i.e.,

$$
\boldsymbol{\pi}_{f}(t-1)=\left(\pi_{f}^{1}(t-1), \cdots, \pi_{f}^{Z_{f}}(t-1)\right) .
$$

Here, we define $\mathbf{r}_{f}(t)=\left(r_{f}^{1}(t), \cdots, r_{f}^{m}(t) \cdots, r_{f}^{Z_{f}}(t)\right)$ as a regret vector of determining flow-split vector for flow $f$. The
MBS selects the flow-split vector with highest regret in which the mixed-strategy probability is given as

$$
\pi_{f}^{m}(t)=\frac{\left[r_{f}^{m}(t)\right]^{+}}{\sum_{m^{\prime} \in \mathcal{Z}_{f}}\left[r_{f}^{m^{\prime}}(t)\right]^{+}} .
$$

Let $\hat{\mathbf{r}}_{f}(t)=\left(\hat{r}_{f}^{1}(t), \cdots, \hat{r}_{f}^{m}(t) \cdots, \hat{r}_{f}^{Z_{f}}(t)\right)$ be the estimated regret vector of flow $f$, we introduce the Boltzmann-Gibbs (BG) distribution, $\boldsymbol{\beta}_{f}^{m}\left(\hat{\mathbf{r}}_{f}(t)\right)$ to capture the exploitation and exploration for efficient learning, given by

$$
\begin{aligned}
\boldsymbol{\beta}_{f}^{m}\left(\hat{\mathbf{r}}_{f}(t)\right)=\underset{\pi_{f} \in \Pi}{\operatorname{argmax}} & \sum_{m \in \mathcal{Z}_{f}}\left[\pi_{f}^{m}(t) \hat{r}_{f}^{m}(t)\right. \\
& \left.-\kappa_{f} \pi_{f}^{m}(t) \ln \left(\pi_{f}^{m}(t)\right)\right],
\end{aligned}
$$

where the trade-off factor $\kappa_{f}$ is used to balance between exploration and exploitation [23], [24]. If $\kappa_{f}$ is small, the MBS selects $\mathbf{z}_{f}$ with highest payoff. For $\kappa_{f} \rightarrow \infty$ all decisions have equal probability.

For a given set of $\hat{\mathbf{r}}_{f}(t)$ and $\kappa_{f}$, we solve (19) to find the probability distribution in which the solution determining the disjoint paths for each flow $f$ is given as

$$
\beta_{f}^{m}\left(\hat{\mathbf{r}}_{f}(t)\right)=\frac{\exp \left(\frac{1}{\kappa_{f}}\left[\hat{r}_{f}^{m}(t)\right]^{+}\right)}{\sum_{m^{\prime} \in \mathcal{Z}_{f}} \exp \left(\frac{1}{\kappa_{f}}\left[\hat{r}_{f}^{m^{\prime}}(t)\right]^{+}\right)} .
$$

We denote $\hat{u}(t)$ as the estimated utility of flow $f$ at time instant $t$ with action $\mathbf{z}_{f}$, i.e, $\hat{\mathbf{u}}_{f}(t)=$ $\left(\hat{u}_{f}^{1}(t), \cdots, \hat{u}_{f}^{m}(t) \cdots, \hat{u}_{f}^{Z_{f}}(t)\right)$. Upon receiving the feedback, $\tilde{u}_{f}(t)$ denotes the utility observed by flow $f$, i.e., $\tilde{u}_{f}(t)=u_{f}(t-1)$. Finally, we propose the learning mechanism at each time instant $t$ as follows.

Learning procedure: The estimates of the utility, regret, and probability distribution functions are performed, and are updated for all actions as follows:

$$
\left\{\begin{array}{l}
\hat{u}_{f}^{m}(t)=\hat{u}_{f}^{m}(t-1)+\xi_{f}(t) \mathbb{I}_{\left\{\mathbf{z}_{f}=\mathbf{z}_{f}^{m}\right\}}\left(\tilde{u}_{f}(t)-\hat{u}_{f}^{m}(t-1)\right), \\
\hat{r}_{f}^{m}(t)=\hat{r}_{f}^{m}(t-1)+\gamma_{f}(t)\left(\hat{u}_{f}^{m}(t)-\tilde{u}_{f}(t)-\hat{r}_{f}^{m}(t-1)\right), \\
\pi_{f}^{m}(t)=\pi_{f}^{m}(t-1)+\iota_{f}(t)\left(\beta_{f}^{m}\left(\hat{\mathbf{r}}_{f}(t)\right)-\pi_{f}^{m}(t-1)\right),
\end{array}\right.
$$

Here, $\xi_{f}(t), \gamma_{f}(t)$, and $\iota_{f}(t)$ are the learning rates which are chosen to satisfy the convergence properties [7]. Based on the probability distribution as per (21), the MBS determines the flow-split vector for each flow $f$ as defined in Section III. Note that the learning-aided path selection is performed in a longterm period to ensure that the paths do not suddenly change such that the SCBSs have enough time to release traffic from the queues. 


\section{B. Rate Allocation}

Consider $R_{(i, j)}^{f}=\log \left(1+p_{(i, j)}^{f}\left|g_{(i, j)}(\mathbf{h})\right|^{2}\right)$ as the transmission rate, where the effective channel gain ${ }^{3}$ for mmWave channels can be modeled as $g_{(i, j)}(\mathbf{h})=\frac{\tilde{g}_{(i, j)}(\mathbf{h})}{1+I_{\max }}$ [25], [4]. Here, $\tilde{g}_{(i, j)}(\mathbf{h})$ and $I^{\max }$ denote the normalized channel gain and the maximum interference, respectively. Denoting the left hand side (LHS) of (10) and (11) as $D_{i}^{f}$ for simplicity, the optimal values of flow control $\mathbf{x}$ and transmit power $\mathbf{p}$ are found by minimizing

$$
\min _{\mathbf{x}, \mathbf{p} \mid \boldsymbol{\pi}} \sum_{f=1}^{F}-Y_{f} x_{f}
$$

subject to $1+p_{\left(i, i_{f}^{(o)}\right)}^{f}\left|g_{\left(i, i_{f}^{(\circ)}\right)}\right|^{2} \geq e^{x_{f}}, \forall f \in \mathcal{F}, i=0$,

$$
\begin{aligned}
& \frac{1+p_{\left(i, i_{f}^{(\mathrm{o})}\right)}^{f}\left|g_{\left(i, i_{f}^{(\mathrm{o})}\right)}\right|^{2}}{1+p_{\left(i_{f}^{(\mathrm{I})}, i\right)}^{f}\left|g_{\left(i_{f}^{(\mathrm{I})}, i\right)}\right|^{2}} \geq e^{D_{i}^{f}}, \forall i \in \mathcal{N}_{i}^{(o)}, f \in \mathcal{F}, \\
& \sum_{f \in F} p_{\left(i, i_{f}^{(o)}\right)}^{f} \leq P_{i}^{\max }, \forall i \in \mathcal{B}, \forall f \in \mathcal{F} .
\end{aligned}
$$

The constraint (22c) is non-convex, but the LHS of (22c) is an affine-over-affine function, which is jointly convex w.r.t the corresponding variables [13]. In this regard, we introduce the slack variable $y$ to $(22 \mathrm{c})$ and rewrite it as

$$
\begin{aligned}
& \frac{2+p_{\left(i, i_{f}^{(\mathrm{o})}\right)}^{f}\left|g_{\left(i, i_{f}^{(\mathrm{o})}\right)}\right|^{2}}{2} \geq \sqrt{y^{2}+\left(\frac{p_{\left(i, i_{f}^{(\circ)}\right)}^{f}\left|g_{\left(i, i_{f}^{(\circ)}\right)}\right|^{2}}{2}\right)^{2}}, \\
& \frac{y^{2}}{1+p_{\left(i_{f}^{(\mathrm{I})}, i\right)}^{f}\left|g_{\left(i_{f}^{(\mathrm{I})}, i\right)}\right|^{2}} \geq e^{D_{i}^{f}}
\end{aligned}
$$

Here, the constraint (23) holds a form of the second-order cone inequalities [13], [26], while the LHS of constraint (24) is a quadratic-over-affine function which is iteratively replaced by the first order to achieve a convex approximation as follow

$$
\frac{2 y y^{(l)}}{1+p_{\left(i_{f}^{(\mathrm{I})}, i\right)}^{f(l)}\left|g_{\left(i_{f}^{(\mathrm{I})}, i\right)}\right|^{2}}-\frac{y^{(l) 2}\left(1+p_{\left(i_{f}^{(\mathrm{I})}, i\right)}^{f}\left|g_{\left(i_{f}^{(\mathrm{I})}, i\right)}\right|^{2}\right)}{\left(1+p_{\left(i_{f}^{(\mathrm{I})}, i\right)}^{f(l)}\left|g_{\left(i_{f}^{(\mathrm{I})}, i\right)}\right|^{2}\right)^{2}} \geq e^{D_{i}^{f}} .
$$

Here, the superscript $l$ denotes the $l$ th iteration. Hence, we iteratively solve the approximated convex problem of (22) as Algorithm 1 in which the approximated problem is given as

$$
\min _{\mathbf{x}, \mathbf{p} \mid \boldsymbol{\pi}} \sum_{f=1}^{F}-Y_{f} x_{f}
$$

$$
\text { subject to (22d), (5), (22b), (23), (25). }
$$

\footnotetext{
${ }^{3}$ The effective channel gain captures the path loss, channel variations, and interference penalty (Here, the impact of interference is considered small due to highly directional beamforming and high pathloss for interfered signals at mmWave frequency band, and thus a multi-hop directional transmission can be operated at dense mmWave networks).
}

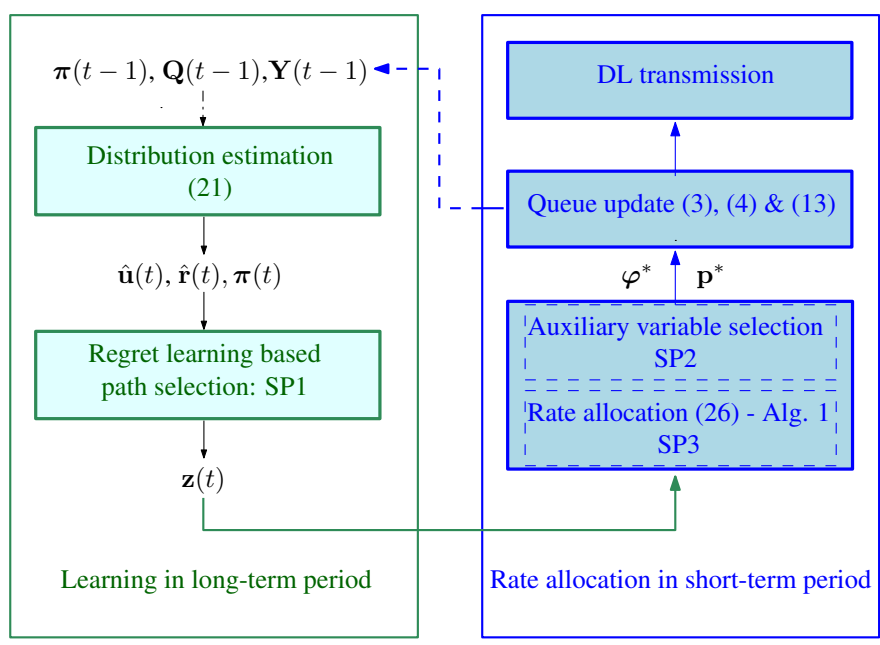

Fig. 2: Information flow diagram of the learning-aided path selection and rate allocation.

Finally, the information flow diagram of the learning-aided path selection and rate allocation approach is shown in Fig. 2, where the rate allocation is executed in a short-term period. Note that the path selection and rate allocation are both done at the MBS, in this work we assume that the information is shared among the base stations by using the $\mathrm{X} 2$ interface.

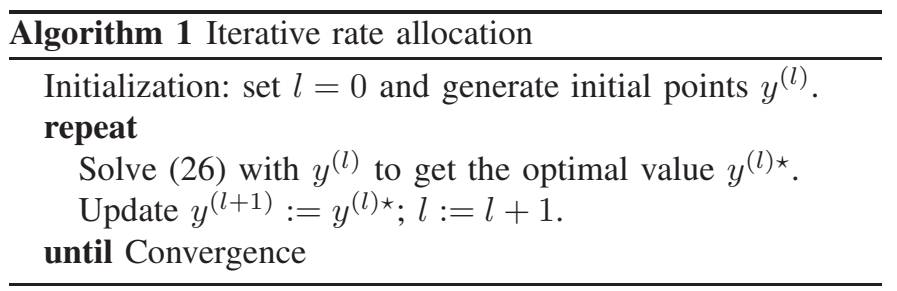

Finally, the information flow diagram of the learning-aided path selection and rate allocation approach is shown in Fig. 2, where the rate allocation is executed in a short-term period. The reason why we chose an iterative method to solve the non-convex optimization problem (22) due to that in general speaking, there is no fast and cost-efficient approach to solve (22). Besides, finding the globally optimal solution for problem (22) via a brute-force approach entails a prohibitively high computational complexity. Hence, we propose an iterative algorithm to obtain an efficient suboptimal solution.

\section{Numerical Results}

We provide numerical results by assuming two flows from the MBS to two UEs, while the number of available paths for each flow is four [10]. The MBS selects two routes from four most popular routes ${ }^{4}$. Each route contains two relays, the total number of SCBSs is 8 , and the one-hop distance is varying from 50 to 100 meters. The maximum transmit power of MBS and each SCBS are $43 \mathrm{dBm}$ and $30 \mathrm{dBm}$, respectively. The

\footnotetext{
${ }^{4}$ As studied in [10], it suffices for a flow to maintain at least two paths provided that it repeatedly selects new paths at random and replaces if the latter provides higher throughput.
} 
SCBS antenna gain is $5 \mathrm{dBi}$ and the number of antennas at each BS is $N_{b}=8$. We assume that the traffic flow is divided equally into two subflows, the arrival rate for each sub-flow is varying from 2 to $5 \mathrm{Gbps}$. The path loss is modeled as a distance-based path loss with the line-of-sight (LOS) model for urban environments at $28 \mathrm{GHz}$ with $1 \mathrm{GHz}$ of bandwidth [4]. The maximum delay requirement $\beta$ and the target reliability probability $\epsilon$ are set to be $10 \mathrm{~ms}$ and 0.05 , respectively [5]. For the learning algorithm, the Boltzmann temperature $\kappa_{f}$ is set to 5 , while the learning rates $\xi_{f}(t), \gamma_{f}(t)$, and $\iota_{f}(t)$ are set to $\frac{1}{(t+1)^{0.5}}, \frac{1}{(t+1)^{0.55}}$, and $\frac{1}{(t+1)^{0.6}}$, respectively [23].

Furthermore, we compare our proposed scheme with the following baselines:

- Baseline 1 considers a general NUM framework [12] with best path learning [23].

- Baseline 2 considers a general NUM framework [12] and a random path section scheme, subject to (6b).

- Baseline 3 considers a general NUM framework [12] and a random path section scheme.

- Single hop scheme: The MBS delivers data to UEs over one single hop at long distance in which the probability of LOS communication is low, and blockage is taken into account.

In Fig. 3, we report the average one-hop delay ${ }^{5}$ versus the mean arrival rates $\bar{\mu}$. As we increase $\bar{\mu}$, baseline 3 violates the latency constraints, whereas our proposed algorithm outperforms the other baselines. The reason behind this gain is that the delay requirement is satisfied via the equivalent instantaneous rate by our proposed algorithm as per (10) and (11), while the baselines $\mathbf{1}$ and $\mathbf{3}$ use the traditional utilitydelay trade-off approach. Moreover, we apply the learning path algorithm, which selects the path with high payoff and less congestion resulting in small delay. The average one-hop delay of baseline 1 with learning outperforms baselines 2 and 3, whereas our proposed scheme reduces latency by $50.64 \%$, $81.32 \%$ and $92.9 \%$ as compared to baselines $\mathbf{1}, \mathbf{2}$, and $\mathbf{3}$, respectively, when $\lambda=4.5 \mathrm{Gbps}$. When $\lambda=5 \mathrm{Gbps}$, the average delay of all baselines increases, violating the delay requirement of $10 \mathrm{~ms}$, while our proposed scheme is robust to the latency requirement. Moreover, for throughput comparison, we observe that for $\lambda=4.5 \mathrm{Gbps}$, our proposed algorithm is able to deliver $4.4874 \mathrm{Gbps}$ of average network throughput per each subflow, while the baselines 1, 2, and $\mathbf{3}$ deliver 4.4759, 4.4682, and 4.3866 Gbps, respectively. Here, the single hop scheme only delivers 3.55 Gbps due to the blockage, which resulting in large delay.

In Fig. 4, we report the tail distribution (complementary cumulative distribution function (CCDF)) of latency to showcase how often the system achieves a delay greater than the target delay levels. In contrast to the average delay, the tail distribution is an important metric to reflect the URLLC characteristic. For instance, at $\lambda=4.5 \mathrm{Gbps}$, by imposing the probabilistic latency constraint, our proposed approach ensures

\footnotetext{
${ }^{5}$ The average end-to-end delay can be defined as the sum of the average one-hop delay of all hops.
}

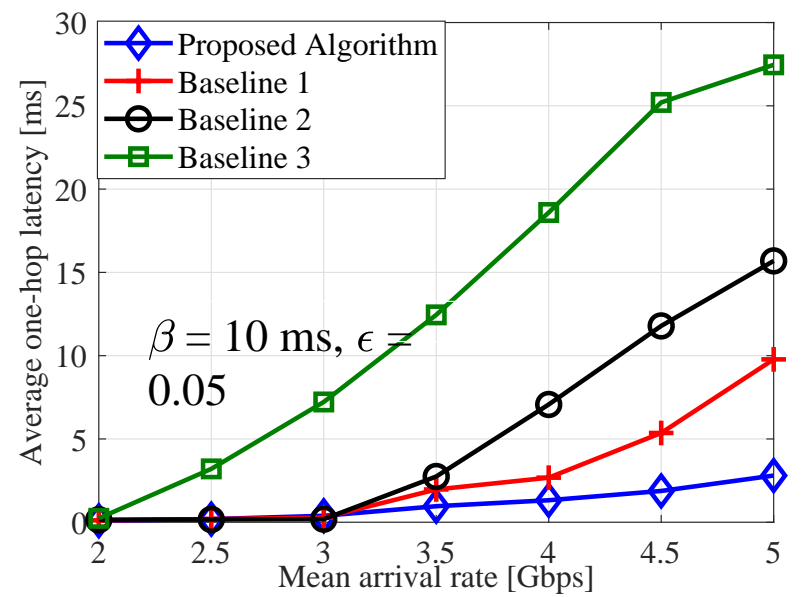

Fig. 3: Average one-hop delay versus mean arrival rates.

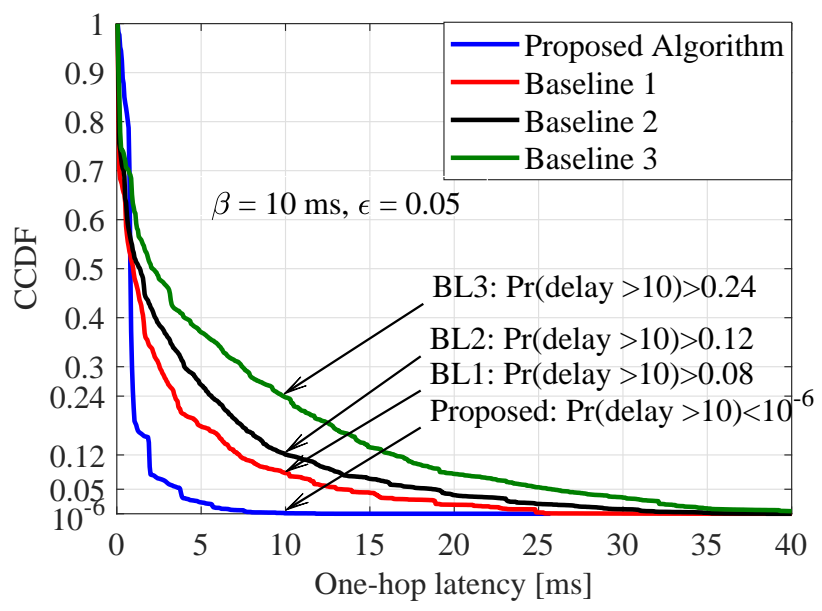

Fig. 4: CCDF of the one-hop latency, $\bar{\mu}=4.5$ Gbps.

reliable communication with better guaranteed probability, i.e, $\operatorname{Pr}($ delay $>10 \mathrm{~ms})<10^{-6}$. In contrast, baseline 1 with learning violates the latency constraint with high probability, where $\operatorname{Pr}($ delay $>10 \mathrm{~ms})=0.08$ and $\operatorname{Pr}($ delay $>25 \mathrm{~ms})<10^{-6}$, while the performance of baselines 2 and $\mathbf{3}$ gets worse.

\section{CONCLUSION}

In this paper, we have proposed a multi-hop scheduling to support reliable communication by incorporating the probabilistic latency constraint in $5 \mathrm{G}$ self-backhauled mmWave networks. In particular, the problem is modeled as a network utility maximization subject to a bounded latency constraint with a guaranteed probability, and queue stability. We have proposed a dynamic approach, which adapts to channel variations and system dynamics. We have leveraged stochastic optimization to decouple the studied problem into path selection and rate allocation sub-problems. Numerical results show that our proposed framework reduces latency by $50.64 \%$ and $92.9 \%$ as compared to baselines.

\section{ACKNOWLEDGMENT}

The authors would like to thank Tekes, Nokia, Huawei, MediaTek, Keysight, Bittium and Kyynel for project funding. 
The Academy of Finland funding via the grant 307492 and the CARMA grants 294128 and 289611, the Nokia Foundation, the Riitta and Jorma J. Takanen Foundation SR grant, the Tauno Tönning Foundation, and the Finnish Technological Promotion Foundation are also acknowledged.

\section{REFERENCES}

[1] J. G. Andrews et al., "What Will 5G Be?" IEEE Journal on Selected Areas in Communications, vol. 32, no. 6, pp. 1065-1082, June 2014.

[2] T. S. Rappaport et al., "Millimeter wave mobile communications for 5G cellular: It will work!" IEEE Access, vol. 1, pp. 335-349, 2013.

[3] A. Anpalagan, M. Bennis, and R. Vannithamby, Design and Deployment of Small Cell Networks. Cambridge University Press, 2015.

[4] T. K. Vu, M. Bennis, S. Samarakoon, M. Debbah, and M. Latva-aho, "Joint load balancing and interference mitigation in $5 \mathrm{G}$ heterogeneous networks," IEEE Transactions on Wireless Communications, vol. 16, no. 9, pp. 6032-6046, Sep. 2017.

[5] T. K. Vu, C.-F. Liu, M. Bennis, M. Debbah, M. Latva-aho, and C. S. Hong, "Ultra-reliable and low latency communication in mmwaveenabled massive MIMO networks," IEEE Communications Letters, vol. 21, no. 9, pp. 2041-2044, Sep. 2017.

[6] S. Singh, F. Ziliotto, U. Madhow, E. Belding, and M. Rodwell, "Blockage and directivity in $60 \mathrm{GHz}$ wireless personal area networks: From cross-layer model to multihop MAC design," IEEE Journal on Selected Areas in Communications, vol. 27, no. 8, 2009.

[7] S. Samarakoon, M. Bennis, W. Saad, and M. Latva-aho, "Backhaulaware interference management in the uplink of wireless small cell networks," IEEE Transactions on Wireless Communications, vol. 12, no. 11, pp. 5813-5825, 2013.

[8] N. Eshraghi et al., "Millimeter-wave device-to-device multi-hop routing for multimedia applications," in Proc. IEEE Int. Conf. Commun., Kuala Lumpur, Malaysia, May 2016, pp. 1-6.

[9] B. Sahoo, C.-H. Yao, and H.-Y. Wei, "Millimeter-wave multi-hop wireless backhauling for $5 \mathrm{~g}$ cellular networks," in Proc. IEEE 85th Vehicular Technology Conf., Sydney, Australia, June 2017, pp. 1-6.

[10] P. Key et al., "Path selection and multipath congestion control," in Proc. the 26th IEEE Int. Conf. on Computer Communications (INFOCOM). Barcelona, Spain: IEEE, 2007, pp. 143-151.

[11] M. Bennis, M. Debbah, and H. V. Poor, "Ultra-Reliable and LowLatency Wireless Communication: Tail, Risk and Scale," submitted to Proceedings of the IEEE, 2018.

[12] M. J. Neely, "Stochastic network optimization with application to communication and queueing systems," Synthesis Lectures on Cоттиnication Networks, vol. 3, no. 1, pp. 1-211, 2010.

[13] S. Boyd and L. Vandenberghe, Convex optimization. Cambridge university press, 2004

[14] T. K. Vu, M. Bennis, S. Samarakoon, M. Debbah, and M. Latvaaho, "Joint in-band backhauling and interference mitigation in $5 \mathrm{G}$ heterogeneous networks," in Proc. 22th European Wireless Conf., Oulu, Finland, May 2016, pp. 1-6.

[15] A. Zakrzewska et al., "Dual connectivity in LTE HetNets with split control-and user-plane," in Proc. IEEE Global Commun. Conf. Workshops, Atlanta, GA, USA, Dec. 2013, pp. 391-396.

[16] J. Liu and E. S. Bentley, "Hybrid-beamforming-based millimeter-wave cellular network optimization," in Proc. 15th International Symposium on Modeling and Optimization in Mobile, Ad Hoc, and Wireless Networks (WiOpt), 2017, pp. 1-8.

[17] J. Wildman et al., "On the joint impact of beamwidth and orientation error on throughput in directional wireless Poisson networks," IEEE Transactions on Wireless Communications, vol. 13, no. 12, pp. 7072 7085, 2014

[18] T. L. Marzetta and B. M. Hochwald, "Fast transfer of channel state information in wireless systems," IEEE Transactions on Signal Processing, vol. 54 , no. 4, pp. 1268-1278, 2006.

[19] J. Palacios et al., "Tracking mm-wave channel dynamics: Fast beam training strategies under mobility," in Proc. 36th Annual IEEE Int. Conf. on Computer Communications (INFOCOM), Atlanta, GA, USA, 2017, pp. $1-9$.

[20] J. D. Little and S. C. Graves, "Little's law," in Building intuition. Springer, 2008, pp. 81-100.
[21] A. Mukherjee, "Queue-aware dynamic on/off switching of small cells in dense heterogeneous networks," in Proc. IEEE Global Commun. Conf. Workshops, Atlanta, GA, USA, Dec. 2013, pp. 182-187.

[22] L. Huang, "Receding learning-aided control in stochastic networks," Perform. Eval., vol. 91, no. C, pp. 150-169, Sep. 2015.

[23] M. Bennis, S. M. Perlaza, and M. Debbah, "Learning coarse correlated equilibria in two-tier wireless networks," in Proc. IEEE Int. Conf Commun., Ottawa, ON, Canada, Jun. 2012, pp. 1592-1596.

[24] S. M. Perlaza, H. Tembine, S. Lasaulce, and M. Debbah, "Quality-ofservice provisioning in decentralized networks: A satisfaction equilibrium approach," IEEE Journal of Selected Topics in Signal Processing, vol. 6, no. 2, pp. 104-116, 2012.

[25] S. Hur et al., "Millimeter wave beamforming for wireless backhaul and access in small cell networks," IEEE Trans. Commun., vol. 61, no. 10, pp. 4391-4403, Oct. 2013.

[26] A. Ben-Tal and A. Nemirovski, Lectures on modern convex optimization: analysis, algorithms, and engineering applications. SIAM, 2001. 